\title{
Plasmodium ovale curtisi and Plasmodium ovale wallikeri in North-West Ethiopia
}

\author{
Abebe Alemu ${ }^{*}$, Hans-Peter Fuehrer ${ }^{2}$, Gebeyaw Getnet ${ }^{1}$, Belay Tessema ${ }^{3}$ and Harald Noedl ${ }^{4}$
}

\begin{abstract}
Background: In Ethiopia Plasmodium falciparum and Plasmodium vivax are the dominant species accounting for roughly 60 and $40 \%$ of malaria cases, respectively. Recently a major shift from $P$. falciparum to $P$. vivax has been observed in various parts of the country but the epidemiology of the other human malaria species, Plasmodium ovale spp. and Plasmodium malariae remains poorly understood. The aim of this study was to assess $P$. ovale curtisi and wallikeri infection in north-west Ethiopia by using microscopy and nested PCR.
\end{abstract}

Methods: A health institution-based survey using non-probability sampling techniques was conducted at Maksegnet, Enfranze and Kola Diba health centres and Metema hospital in North Gondar. Three-hundred patients with signs and symptoms consistent with malaria were included in this study and capillary blood was collected for microscopic examination and molecular analysis of Plasmodium species. Samples were collected on Whatman 903 filter papers, stored in small plastic bags with desiccant and transported to Vienna (Austria) for molecular analysis. Data from study participants were entered and analysed by SPSS 20 software.

Results: Out of 300 study participants (167 males and 133 females), 184 samples were classified positive for malaria (133 P. falciparum and 51 P. vivax) by microscopy. By species-specific PCR 233 Plasmodium spp (95\% Cl: 72.6-82) were detected and the majority 155 (66.5\%, 95\% Cl: 60.2-72.3) were $P$. falciparum followed by $P$. vivax 69 (29.6\%, 95\% Cl; 24.1-35.8) and 9 (3.9\%, 95\% Cl: 2-7.2) samples were positive for $P$. ovale. Seven of $P$. ovale parasites were confirmed as $P$. ovale wallikeri and two were confirmed as $P$. ovale curtisi. None of the samples tested positive for P. malariae. During microscopic examination there were high (16.3\%) false negative reports and all mixed infections and $P$. ovale cases were missed or misclassified.

Conclusion: This study indicates that $P$. ovale malaria is under-reported in Ethiopia and provides the first known evidence of the sympatric distribution of indigenous P. ovale wallikeri and P. ovale curtisi in Ethiopia. Therefore, further studies assessing the prevalence of the rare species $P$. ovale and $P$. malariae are urgently needed to better understand the species distribution and to adapt malaria control strategies.

\section{Background}

Malaria remains one of the leading causes of illness and death in the world [1,2]. Plasmodium ovale (first described by Stevens in 1922) [3] is widely distributed across tropical regions in Africa and Asia and is one out of five Plasmodium parasite species that causes human malaria $[4,5]$. Relatively little attention has been paid to ovale malaria, which is considered to be uncommon,

\footnotetext{
* Correspondence: yanbule@gmail.com

'Department of Medical Parasitology, School of Biomedical and Laboratory Sciences, College of Medicine and Health Sciences, University of Gondar, Gondar, Ethiopia

Full list of author information is available at the end of the article
}

mild in clinical presentation and easily treated with the conventional anti-malarial chloroquine [6].

In a study on $P$. ovale conducted by researchers from the UK Malaria Reference Laboratory, London, polymorphisms in six loci were examined in 55 isolates. Two distinct major haplotypes of each locus were identified and these did not recombine in any of the parasites examined. Accordingly, P. ovale dimorphism was proposed to reflect the existence of two fully distinct ovale malaria species, which were unexpectedly shown to be broadly sympatric, at the country level, in both Africa and Asia. These two proposed species have been named P. ovale curtisi and P. ovale wallikeri [7]. Recently both ovale species have been identified in a single individual

\section{Biomed Central}

(c) 2013 Alemu et al.; licensee BioMed Central Ltd. This is an Open Access article distributed under the terms of the Creative Commons Attribution License (http://creativecommons.org/licenses/by/2.0), which permits unrestricted use, distribution, and reproduction in any medium, provided the original work is properly cited. 
further strengthening the concept of two distinct $P$. ovale species $[8,9]$.

Malaria remains the leading communicable disease in Ethiopia, accounting for approximately $30 \%$ of the overall disability-adjusted life years lost. It is estimated that about $75 \%$ of the total area of the country and $68 \%$ of the population is at risk of infection [10-12]. In the country Plasmodium falciparum and Plasmodium vivax are the main species accounting for roughly 60 and $40 \%$ of malaria cases [13] and recently there has been a major shift from $P$. falciparum to $P$. vivax in different parts of the country. Despite First report on P. ovale in Ethiopia by Armstrong JC IN 1969 [14], the knowledge of the distribution and epidemiology of the other human malaria species, particularly $P$. ovale spp. and Plasmodium malariae has never been elucidated and is rather limited in the entire country [15-17].

The global burden of ovale malaria is considered to be light, potentially also because $P$. ovale tends to be misand under-diagnosed when using microscopic examination. Difficulties in the diagnosis are partly the result of the low parasite density that is characteristic of malaria caused by ovale malaria parasites $[6,18,19]$. Species determination at low parasite density is particularly difficult and largely depends on individual skill [20,21]. In developing countries, including Ethiopia, where malaria is diagnosed microscopically by staining thick and thin blood films with Giesma (which despite its inherent limitations remains the gold standard) and where few other diagnostic options are available, $P$. ovale malaria tends to be under-reported.

Therefore, new laboratory diagnostic techniques (such as polymerase chain reaction (PCR)) that can provide higher sensitivity and specificity, without subjective variation, are urgently needed to improve the quality of diagnostics and the understanding of malaria epidemiology [22-24]. The main focus of this study was to assess $P$. ovale species and the ability of microscopists to detect and identify Plasmodium species in north-west Ethiopia.

\section{Methods}

\section{Study area}

The study was conducted at three health centres (Maksegnet, Enfranze and Kola Diba) and one hospital (Metema hospital) in North Gondar, northwest Ethiopia. These areas are endemic for malaria and the altitude of the districts ranges from 1,750 to $2100 \mathrm{~m}$ above sea level. Malaria is the most prevalent seasonal disease in the area, ranking second among all reported diseases seen at the health centres. October to December is the peak transmission season for $P$. falciparum. Both $P$. vivax and $P$. falciparum are known to co-exist in the area whereas other Plasmodium species have not been reported in the past two decades from any of the four sites. The only reports of $P$. ovale in Ethiopia date back to the 1960s [14].

\section{Study design and inclusion criteria}

A health institution-based survey using non-probability sampling techniques was conducted at three health centres and one hospital in North Gondar, north-west Ethiopia. Patients with signs and symptoms consistent with malaria (for which diagnostic blood films are requested at any of the participating health institutions) were included in this study. Males and females, of any age, with signs and symptoms consistent with malaria and willingness to participate and sign the informed consent, were used as inclusion criteria.

\section{Sample size and sampling techniques}

Convenience sampling technique was used to recruit study participants for this study. A total of 300 suspected malaria patients were included from March 2 to April 26, 2013. Participants were 184 microscopy-confirmed cases and 116 febrile patients suspected of potentially having malaria and who were negative by microscopy. A structured questionnaire was used to obtain sociodemographic information (sex, age, residence, ethnicity, religion, and clinical data).

\section{Blood sample collection and microscopic malaria parasite examination}

From each participant a small amount of capillary blood was collected by finger prick after obtaining informed consent. The total amount of blood was less than $1 \mathrm{~mL}$. Peripheral smear examination of well-prepared and wellstained blood films remains the gold standard for confirming the presence of malaria parasites. Thick and thin smears prepared from the peripheral blood were used for this purpose and for each patient two slides were prepared.

Before staining the thin blood films were fixed in methanol for $30 \mathrm{sec}$. Then smears used for study purposes were stained with $3 \%$ Giemsa solution for $30 \mathrm{~min}$, whereas the staining of the routine smear followed the standard techniques established at the respective health facility. Examination of the blood films was conducted according to standardized World Health Organization (WHO) protocols. Thick films were used for the detection of the presence of parasites and parasite species identification was done by using thin films under $100 \times$ oil immersion objective.

\section{Sample collection for molecular analysis}

Two blood spots were collected from each participant, transferred onto filter paper (Whatman 903) and labelled with the participant's study code and date. Each filter paper was dried individually, carefully avoiding any chance 
of contamination. The samples were then stored in small plastic bags with desiccant and transported to the Institute of Specific Prophylaxis and Tropical Medicine, Medical University of Vienna (MUV), Vienna, Austria for molecular analysis.

\section{DNA isolation and purification}

A modified chelex-based DNA extraction method using the InstaGene Whole Blood Kit (Bio-Rad Laboratories, Hercules, CA, USA) was used for the extraction and purification of Plasmodium DNA from the blood spots on filter paper. To ensure comparable quantities of blood, samples of exactly $4 \mathrm{~mm}$ diameter were punched out of the blood spots. Blood spots were soaked overnight in a tube at $4^{\circ} \mathrm{C}$ with $100 \mu$ l phosphate-buffered saline (PBS). The tube was centrifuged at 13,000 revolutions per minute (rpm) for $2 \mathrm{~min}$. After discarding the supernatant, $100 \mu \mathrm{L}$ of PBS was added to the tube and the sample was centrifuged at $13,000 \mathrm{rpm}$ for $2 \mathrm{~min}$ to wash the sediment. After discarding the supernatant, $60 \mu \mathrm{L}$ of InstaGene Matrix was added. The tube was incubated for $8 \mathrm{~min}$ at $70^{\circ} \mathrm{C}$, vortexed for $15 \mathrm{sec}$, boiled for $4 \mathrm{~min}$, and vortexed for $15 \mathrm{sec}$. Finally, the tube was centrifuged at 13,000 rpm for $1 \mathrm{~min}$ and the supernatant was aspirated and purified again with InstaGene Matrix as described. The supernatant was then used for the PCR. If the PCR result was negative, the supernatant was purified again for up to three times.

\section{Parasite detection by nested PCR}

Nested PCR assay was performed for all samples as reported previously [22,24-26]. Each $50 \mu \mathrm{l}$ reaction mixture for nest 1 amplifications contained $5 \mu \mathrm{l}$ of DNA template, $5 \mu \mathrm{l}(10 \mu \mathrm{M})$ of each primer (rPLU 1 and rPLU 5), $2 \mathrm{mM}$ $\mathrm{MgCl}_{2}$, PCR buffer, $125 \mu \mathrm{M}$ of each dNTP, and 1 unit of GoTaq DNA polymerase (Promega, Madison, WI, USA). Nest 1 amplification conditions were as follows: initial denaturation $94^{\circ} \mathrm{C}$ for $4 \mathrm{~min} ; 25$ cycles $(1 \mathrm{~min}$ denaturation at $94^{\circ} \mathrm{C}$, annealing at $58^{\circ} \mathrm{C}$ for $2 \mathrm{~min}$, extension at $72^{\circ} \mathrm{C}$ for $2 \mathrm{~min}$ ); and a final extension at $72^{\circ} \mathrm{C}$ for $4 \mathrm{~min}$; $2.5 \mu \mathrm{l}$ of the nest 1 amplification product served as the DNA template for each of the $25 \mu \mathrm{l}$ nest 2 amplifications.

The concentration of the nest 2 primers and other constituents were identical to nest 1 amplifications. Nest 2 amplification conditions were identical for all speciesspecific nest 2 PCRs to those of nest 1 except for the genus-specific primers (rPLU 3 and 4; annealing: $62^{\circ} \mathrm{C}$ ). In all experiments, a negative control of water and known positive controls were run with the samples. In the nested PCR, the genus-specific primers rPLU1 and rPLU5 for the first PCR and rPLU3 and rPLU4 were used for the second PCR. Whenever this genus-specific nested PCR gave positive results, species-specific nest 2 PCRs were performed for species determination using the following internal primer pairs: rFAL1 and rFAL2 for P. falciparum, rVIV1 and rVIV2 for P. vivax, rMAL1 and rMAL2 for $P$. malariae, rOVA1WC and rOVA2WC for both $P$. ovale species [22,24-26].

The oligonucleotides were obtained from Microsynth (Microsynth AG, Balgach, Switzerland). All samples giving positve signals for $P$. ovale spp were further analysed to species level using the primers rOVA1/rOVA2 specific for Plasmodium ovale curtisi and rOVA1v/rOVA2v for Plasmodium ovale wallikeri $[25,26]$. The PCR products of nest 2 amplifications were analysed by gel electrophoresis with $2 \%$ agarose and Midori Green staining. The individual interpreting the PCR results was blinded to the results of microscopy.

\section{Sequence analysis}

The amplicons of $P$. ovale-positive samples were further analysed by sequencing for species confirmation. PCR products were purified with ExoSap-IT ${ }^{\circ}$ (GE Healthcare, Buckinghamshire, UK) and sequenced using the BigDye Sequencing Kit and an automatic 310 ABI PRISM sequencer (PE Applied Biosystems, Weiterstadt, Germany).

\section{Data analysis}

Data were checked for their completeness before entering for analysis; then all the data from study participants were imported into Excel and analysed by SPSS 20 program software. Descriptive statistics was used to assess the distribution of the sociodemographic characteristics and independent variables as well as frequencies and proportion of malaria-positive samples.

\section{Ethical consideration}

This study was expected to pose only minimal risks to the participants. The study protocol was reviewed and approved by the Institutional Ethical Review Board of the University of Gondar. Written informed consent in local language was obtained from each participant and/ or his/her legal representative. For patients who cannot read or write the consent form was read in the local language and a thumbprint was collected instead of a signature. Patients who were positive for malaria parasites received standard treatment according to national treatment guidelines.

\section{Results}

\section{Sociodemography characteristics of the study subjects}

Out of a total of 300 study participants, 167 (55.7\%) were male and 133 (44.3\%) were female. The majority of the respondents (91.7\%) were of Amhara ethnicity and orthodox christianity was the predominant religion in the area accounting for $93.7 \%$. In total $37.3 \%$ of the study participants were illiterate and the majority (61\%) were in the age group 15 to 44 years (Table 1). 
Table 1 Sociodemography characteristics of malariasuspected febrile patients in three health centres and one hospital in North Gondar, northwest Ethiopia, 2013 $(\mathrm{n}=\mathbf{3 0 0})$

\begin{tabular}{|c|c|c|}
\hline Variables & Frequency & Percentage \\
\hline \multicolumn{3}{|l|}{ Sites } \\
\hline Kola Diba & 65 & 21.7 \\
\hline Maksegnet & 72 & 24 \\
\hline Enfranze & 113 & 37.7 \\
\hline Metema & 50 & 16.6 \\
\hline \multicolumn{3}{|l|}{ Gender } \\
\hline Male & 167 & 55.7 \\
\hline Female & 133 & 44.3 \\
\hline \multicolumn{3}{|l|}{ Age } \\
\hline$<5$ & 30 & 10 \\
\hline $5-14$ & 61 & 20.3 \\
\hline $15-44$ & 183 & 61 \\
\hline$>44$ & 26 & 8.7 \\
\hline \multicolumn{3}{|l|}{ Religion } \\
\hline Orthodox & 281 & 93.7 \\
\hline Muslim & 13 & 4.3 \\
\hline Others & 6 & 2 \\
\hline \multicolumn{3}{|l|}{ Ethnicity } \\
\hline Amhara & 275 & 91.7 \\
\hline Tigrea & 21 & 7 \\
\hline Others & 4 & 1.3 \\
\hline \multicolumn{3}{|l|}{ Educational status } \\
\hline Illiterate & 112 & 37.3 \\
\hline Read and write & 100 & 33.3 \\
\hline Elementary completed and above & 88 & 29.4 \\
\hline
\end{tabular}

Comparison of malaria parasite detection by microscopy and nested PCR

A total number of 184 (133 P. falciparum and $51 P$. vivax) samples classified as positive by microscopic examination were confirmed as being positive by PCR. In addition, 49 samples that were classified as negative by microscopy were positive in the genus-specific nested PCR. Only two $(0.7 \%, 95 \%$ CI: $0.2-2.4)$ samples that were read as positive by microscopy were considered false positive. The result indicates that $16.3 \%(49 / 300)$ of the malaria cases were missed by microscopy. By species-specific PCR, 233 Plasmodium spp (95\% CI: 72.6-82) were detected and the majority 155 (66.5\%, 95\% CI: 60.2-72.3) were P. falciparum followed by $P$. vivax 69 (29.6\%, 95\% CI; 24.1-35.8) and nine $(3.9 \%$,) samples were positive for $P$. ovale. Furthermore, nine (3.9\%, 95\% CI: 2-7.2) samples gave positive results for $P$. ovale spp, but all examined samples were negative for P. malariae (Table 2).
All positive samples (184) were mono-infections and all mixed Plasmodium infections were missed and all $P$. ovale were missed or wrongly classified by microscopy. By the PCR results, out of 233 positive samples 15 (6.4\%, 95\% CI: 3.9-10.4) samples were mixed malaria infections, which indicates that the majority of 218 (93.6\%, 95\% CI: 89.7-96.1) cases were mono-infections.

(Table 2) Additionally, PCR analysis revealed 49 (16.3\%, 95\% CI: 12.6-20.9) cases gave false negative results by microscopy and only two $(0.7 \%, 95 \%$ CI: $0.2-2.4)$ false positive reporting of microscopic slides from febrile patients. In addition to false negative and positive report, there was wrong classification of Plasmodium species by microscopy. Three $P$. ovale parasites were reported as $P$. vivax, two were reported as $P$. falciparum, eight $P$. falciparum as $P$. vivax and four $P$. vivax as $P$. falciparum.

\section{Distribution of Plasmodium ovale species among symptomatic patients in North Gondar}

Nine out of 300 (3\%) blood samples collected from symptomatic patients presenting febrile illnesses were positive for $P$. ovale spp, out of which the majority, seven cases (77.7\%) were confirmed as $P$. ovale wallikeri and only two cases $(23.3 \%)$ were confirmed as $P$. ovale curtisi. Four samples positive for $P$. ovale in PCR were confirmed by DNA sequencing (GenBank Accession numbers: KF536873 and KF536874-P. ovale curtisi; KF536875 and KF536876$P$. ovale wallikeri). However, none were diagnosed as $P$. ovale, as $P$. ovale was not known to be endemic in Ethiopia, including in the study areas and, therefore, it was not adequately considered by the microscopists (Table 3). Plasmodium ovale is distributed in all sites in north Gondar with high frequency in Kola Diba.

\section{Discussion}

Malaria remains a leading cause of infectious disease in Ethiopia, accounting for approximately $30 \%$ of the overall disability-adjusted life years lost. It is estimated that about $75 \%$ of the total area of the country and $68 \%$ of the population is at risk of infection. In the country, the two Plasmodium species, P. falciparum and P. vivax, are the main and dominant species accounting for roughly 60 and $40 \%$ of malaria cases, respectively [13]. Recently, there has been a major shift from P. falciparum to $P$. vivax in different parts of the country. However, the epidemiology of the $P$. ovale spp remains poorly understood and there are no recent data on the distribution of this pathogen in Ethiopia (including the study area) [15-17].

The PCR results of this study indicate that $P$. falciparum (66.5\%) remains the predominant species followed by P. vivax $(29.6 \%)$ and P. ovale (3.9\%) at the study sites. The majority (93.6\%) of infections were mono-infections. This result is concordant with previous studies elsewhere in Ethiopia and the report of the Ethiopian Federal Ministry 
Table 2 Comparison of malaria parasite detection by microscopy and nested PCR in three health centres and one hospital in North Gondar, northwest Ethiopia, 2013 ( $n=300$ )

\begin{tabular}{|c|c|c|c|c|c|c|c|c|c|}
\hline \multirow[t]{2}{*}{ Sites } & \multirow[t]{2}{*}{ Total slide examined } & \multicolumn{8}{|c|}{ Microscopically confirmed malaria parasites } \\
\hline & & Pf & Pv & Po & $\mathrm{Pm}$ & $P f+P v$ & $\mathrm{Pf}+\mathrm{Po}$ & $\mathrm{Pv}+\mathrm{Po}$ & $P f+P v+P o$ \\
\hline Kola Diba & 65 & 27 & 9 & - & - & - & - & - & - \\
\hline Maksegnet & 72 & 25 & 11 & - & - & - & - & - & - \\
\hline Enfranze & 113 & 60 & 28 & - & - & - & - & - & - \\
\hline Metema & 50 & 21 & 3 & - & - & - & - & - & - \\
\hline Total & 300 & 133 & 51 & & & & & & \\
\hline \multirow[t]{2}{*}{ Sites } & Total sample analysed & \multicolumn{8}{|c|}{ Result of species specific nested PCR } \\
\hline & & Pf & Pv & Po & $\mathrm{Pm}$ & $P f+P v$ & $\mathrm{Pf}+\mathrm{Po}$ & $P v+P o$ & $P f+P v+P o$ \\
\hline Kola Diba & 65 & 21 & 8 & 3 & - & 6 & 2 & - & - \\
\hline Maksegnet & 72 & 30 & 16 & 2 & - & 2 & - & - & - \\
\hline Enfranze & 113 & 63 & 28 & 1 & - & 3 & - & - & - \\
\hline Metema & 50 & 26 & 5 & - & - & 1 & 1 & - & - \\
\hline Total & 300 & 140 & 57 & & & 12 & 3 & & \\
\hline
\end{tabular}

Note: $\mathrm{Pf}=\mathrm{P}$. falciparum $; \mathrm{Pv}=\mathrm{P}$. vivax $; \mathrm{Pm}=\mathrm{P}$. malariae $; \mathrm{Po}=\mathrm{P}$. ovale.

of Health, in which $P$. falciparum is the predominant malaria circulating in the country followed by $P$. vivax. On the other hand, this finding contradicts previous results, which indicated the shifting of $P$. vivax in the study area and different parts of Ethiopia and no report of $P$. ovale cases recently [15-17]. However, virtually all previous studies conducted in Ethiopia are based on microscopic data only, whereas this study used a highly sensitive nested PCR assay with a documented limit of detection of only six parasites/ $\mu \mathrm{L}$ [27].

The $3.9 \%$ positive rate for $P$. ovale infections among suspected malaria cases is the first report of $P$. ovale from this part of the country. Until relatively recently the distribution pattern of $P$. ovale was considered to be limited to tropical regions in sub-Saharan Africa, Indonesia (e.g., Timor, Flores, West Papua) and the Philippines [6], but not from health facilities treating malaria in Ethiopia, possibly due to its tertian periodicity, typically low parasitaemia and morphological resemblance (when fixed and stained) to P. vivax [28]. In analogy to previous studies, three $P$. ovale cases were misclassified as

Table 3 Distribution of Plasmodium ovale species among symptomatic patients in North Gondar, northwest Ethiopia, $2013(n=300)$

\begin{tabular}{llll}
\hline Sites & $\begin{array}{l}\text { Total samples } \\
\text { analysed }\end{array}$ & \multicolumn{2}{l}{ Plasmodium ovale species } \\
\cline { 3 - 4 } & 65 & P. ovale wallikeri & P. ovale curtisi \\
\hline Kola Diba & 65 & 3 & 2 \\
Maksegnet & 72 & 2 & \\
Enfranze & 113 & 1 & \\
Metema & 50 & 1 & \\
\hline
\end{tabular}

$P$. vivax and two were reported as $P$. falciparum, suggesting difficulty in the diagnosis of $P$. ovale by microscopic examination in the study area where this pathogen was previously not known to be present and most microscopists are neither trained to detect nor aware of the presence of other species [12].

$P$ ovale-positive samples were further analysed with specific PCRs and/or sequenced to classify $P$. ovale species in the study area and the majority $(77.7 \%)$ were confirmed as $P$. ovale wallikeri as compared to $23.3 \%$ that were identified as $P$. ovale curtisi. This finding is in line with the previous report by Sutherland et al. [7], that showed the perfect linkage between the dimorphic forms at each locus and led to the introduction and segregation of $P$. ovale into two species, namely $P$. ovale curtisi (former classic type) and P. ovale wallikeri (former variant type). This finding is the first of its kind in Ethiopia and is in line with previous reports from South Asia in which both $P$. ovale curtisi and $P$. ovale wallikeri occur sympatrically in the Chittagong Hill Tracts, Bangladesh $[29,30]$. These two P. ovale species are morphologically identical and cannot be differentiated by microscopy [31]. Even molecular analysis has previously resulted in inconsistent results [32].

The nested PCR analysis suggests a relatively high false negative rate of $16.3 \%$ with only $0.7 \%$ false positive cases at microscopy as well as misclassification of Plasmodium species. (e.g., P. ovale as P. vivax or P. falciparum; P. falciparum as $P$. vivax; $P$. vivax as $P$. falciparum). Although microscopy remains the gold standard for malaria diagnosis in the field, the limits of detection may significantly differ between microscopists and have previously been estimated to range from 50 to 100 parasites/ $\mu$ l under 
field conditions [23]. Microscopy depends on well maintained equipment, uninterrupted supply of good-quality reagents, trained staff, and good-quality monitoring and supervisory systems. Maintaining a quality-assured microscopy service is a major challenge, even for health centres and district hospitals.

The consequences of misdiagnosis of malaria are felt not only at health centres, but also at national levels. Individuals wrongly diagnosed with malaria are exposed to unnecessary side-effects of drugs, and the true causes are not recognized or treated. This scenario is likely to lead to prolonged and worsening illness with loss of income or productivity, and repeated visits to health providers [21]. This study tried to assess the existence and genetic diversity of $P$. ovale parasites while at the same time assessing the ability of microscopists to detect and identify Plasmodium species. In spite of its limitations (mainly the inability to determine true prevalence), this study assessing of $P$. ovale $s p p$. is the first of its kind in Ethiopia and provides important information regarding the Plasmodium species spectrum and distribution in the country as the basis for larger scale surveys.

\section{Conclusion}

Despite of the high prevalence of malaria in Ethiopia and in the study areas, there had previously been no report of the presence of either P. ovale wallikeri or P. ovale curtisi. On the one hand this study provides $P$. ovale malaria is under-reported and the first known evidence of the sympatric distribution of indigenous $P$. ovale wallikeri and $P$. ovale curtisi in northwest Ethiopia and is meant to provide the Ethiopian government and researchers with guidance regarding the presence of Plasmodium species other than $P$. falciparum and $P$. vivax. At the same time it highlights the importance of adequate training for health staff involved in malaria diagnosis and the inherent limitations of microscopic diagnosis.

\section{Competing interests}

The authors declare that they have no competing interests.

\section{Authors' contributions \\ AA and HN conceived the study. AA and HFP undertook statistical analysis, undertook molecular analysis of the samples and drafted the manuscript. GG and BT contributed in proposal writing, blood sample collection and microscopic diagnosis of malaria slides. All authors contributed to the writing of the manuscript and approved the submitted version of the manuscript.}

\section{Acknowledgements}

We are grateful to MARIB (Malaria Research Initiative Bandarban) for funding this study. The authors wish to thank data collectors at each study site and the study participants for their cooperation in providing the necessary information and blood sample. Furthermore, we wish to thank Ingrid Blöschl and Walpurga Wille-Piazzai for their excellent technical support.

\section{Author details}

'Department of Medical Parasitology, School of Biomedical and Laboratory Sciences, College of Medicine and Health Sciences, University of Gondar,
}

Gondar, Ethiopia. ${ }^{2}$ Department of Pathobiology, Institute of Parasitology, University of Veterinary Medicine Vienna, Vienna, Austria. ${ }^{3}$ Department of Medical Microbiology, School of Biomedical and Laboratory Sciences, College of Medicine and Health Sciences, University of Gondar, Gondar, Ethiopia. ${ }^{4}$ Institute of Specific Prophylaxis and Tropical Medicine, Medical University of Vienna, Vienna, Austria.

Received: 21 August 2013 Accepted: 26 September 2013

Published: 28 September 2013

\section{References}

1. WHO: Expert committee on malaria 20th report: WHO technical report series 892. Geneva: World Health Organization; 2000.

2. MMV website: Curing malaria together. 2008. [http://www.mmv.org], Accessed October 16.

3. Rousset JJ, Couzineau P, Baufine-Ducrocq H: [Plasmodium ovale (Stephens 1922)] (in French). Ann Parasitol Hum Comp 1969, 44:273-328.

4. Martens P, Hall L: Malaria on the move: human population movement and malaria transmission. Emerg Infect Dis 2000, 6:28-45.

5. Rowe AK, Rowe SY, Snow RW: The burden of malaria mortality among African children. Inter J Epid 2006, 35:691-704.

6. Mueller I, Zimmerman PA, Reeder JC: Plasmodium malariae and Plasmodium ovale the "bashful" malaria parasites. Trends Parasitol 2007, 23:278-283.

7. Sutherland CJ, Tanomsing N, Nolder D, Oguike M, Jennison C, Pukrittayakamee S, Dolecek C, Hien TT, Do Rosário VE, Arez AP, Pinto J, Michon P, Escalante AA, Nosten F, Burke M, Lee R, Blaze M, Otto TD, Barnwell JW, Pain A, Williams J, White NJ, Day NP, Snounou G, Lockhart PJ, Chiodini PL, Imwong M, Polley SD: Two nonrecombining sympatric forms of the human malaria parasite Plasmodium ovale occur globally. I Infect Dis 2010, 201:1544-1550.

8. Fuehrer HP, Habler VE, Fally MA, Harl J, Starzengruber P, Swoboda P, Bloeschl I, Khan WA, Noedl H: Plasmodium ovale in Bangladesh: genetic diversity and the first known evidence of the sympatric distribution of Plasmodium ovale curtisi and Plasmodium ovale wallikeri in southern Asia. Int J Parasitol 2012, 42:693-699.

9. Putaporntip C, Hughes AL, Jongwutiwes S: Low level of sequence diversity at merozoite surface protein-1 locus of Plasmodium ovale curtisi and P. ovale wallikeri from Thai isolates. PLoS One 2013, 8:1-8.

10. Ministry of Health: Health and health related indicators: planning and programming department. Addis Ababa, Ethiopia: $\mathrm{FMOH} ; 2004$.

11. Federal Ministry of Health Ethiopia and World Health Organization: Entomological profile of malaria in Ethiopia; 2007.

12. Ministry of Health: Guideline for malaria vector control in Ethiopia: malaria and other vector borne diseases prevention and control team. Addis Ababa: Diseases prevention \& Control Department: MOH; 2002.

13. WHO: World malaria report. Geneva: World Health Organization; 2012.

14. Armstrong JC: Plasmodium ovale endemic in Ethiopia. Trans $R$ Soc Trop Med Hyg 1969, 63:287-288

15. Alemu A, Tesfaye W, Golassa L, Abebe G: Urban malaria and associated risk factors in Jimma town, South-West Ethiopia. Malar J 2011, 10:173.

16. Alemu A, Tesfaye W, Golassa L, Abebe G: Climatic variables and malaria transmission dynamics in Jimma town, South West Ethiopia. Parasit Vectors 2011, 4:30

17. Alemu A, Muluye A, Mihret M, Adugna M, Gebeyaw M: Ten year trend analysis of malaria prevalence in Kola Diba, North Gondar, Northwest Ethiopia. Parasit Vectors 2012, 5:173.

18. Faye FBK, Konaté L, Rogier C, Trape JF: Plasmodium ovale in a highly malaria endemic area of Senegal. Trans R Soc Trop Med Hyg 1998, 92:522-525

19. Faye FB, Spiegel A, Tall A: Diagnostic criteria and risk factors for Plasmodium ovale malaria. J Infect Dis 2002, 186:690-695.

20. World Health Organization: WHO information consultation on recent advances in diagnostic techniques and vaccines for malaria: a rapid dipstick antigen capture assay for the diagnosis of falciparum malaria. Bull World Health Organ 1996, 74:47-54.

21. Bell $D$, Wongsrichanalai $C$, Barnwell JW: Ensuring quality and access for malaria diagnosis: how can it be achieved? Nat Rev Microbiol 2006, 4:7-20.

22. Snounou G, Singh B: Nested PCR analysis of Plasmodium parasites. Methods Mol Med 2002, 72:189-203. 
23. Wongsrichanalai $\mathrm{CM}$, Barcus J, Muth S, Sutamihardja A, Wernsdorfer WH: A review of malaria diagnostic tools: microscopy and rapid diagnostic test (RDT). Am J Trop Med Hyg 2007, 77:119-127.

24. Snounou G, Viriyakosol S, Zhu XP: High sensitivity of detection of human malaria parasites by the use of nested polymerase chain reaction. Mol Biochem Parasitol 1993, 61:315-320.

25. Calderaro A, Piccolo G, Perandin F: Genetic polymorphisms influence Plasmodium ovale PCR detection accuracy. J Clin Microbiol 2007, 45:1624-1627.

26. Fuehrer HP, Stadler MT, Buczolich K, Bloeschl I, Noedl H: Two techniques to simultaneously identify Plasmodium ovale curtisi and Plasmodium ovale wallikeri using the small subunit rRNA gene. J Clin Microbiol 2012, 50:4100-4102.

27. Singh B, Bobogare A, Cox-Singh J, Snounou G, Abdullah MS, Rahman HA: A genus-and species-specific nested polymerase chain reaction malaria detection assay for epidemiological studies. Am J Trop Med Hyg 1999, 60:687-692.

28. Collins WE, Jeffery GM: Plasmodium ovale: parasite and disease. Clin Microbiol Rev 2005, 18:570-581.

29. Fuehrer HP, Starzengruber P, Swoboda P, Khan WA, Matt J, Ley B, Thriemer $K$ Haque R, Yunus EB, Hossain SM, Walochnik J, Noedl H: Indigenous Plasmodium ovale malaria in Bangladesh. Am J Trop Med Hyg 2010, 83:75-78.

30. Oguike MC, Betson M, Burke M, Nolder D, Stothard JR, Kleinschmid I, Proietti C, Bousema T, Ndounga M, Tanabe K, Ntege E, Culleton R, Sutherland CJ: Plasmodium ovale curtisi and Plasmodium ovale wallikeri circulate simultaneously in African communities. Int J Parasitol 2011, 41:677-683.

31. Nolder D, Oguike MC, Maxwell-Scott H, Niyazi HA, Smith V, Chiodini PL, Sutherland CJ: An observational study of malaria in British travellers: Plasmodium ovale wallikeri and Plasmodium ovale curtisi differ significantly in the duration of latency. BMJ Open 2013, 3:e002711. doi: 10.1136/bmjopen-2013-002711.

32. Snounou G, Pinheiro L, Goncalves A, Fonseca L, Dias F: The importance of sensitive detection of malaria parasites in the human and insect hosts in epidemiological studies, as shown by the analysis of field samples from Guinea Bissau. Trans R Soc Trop Med Hyg 1993, 87:649-653.

doi:10.1186/1475-2875-12-346

Cite this article as: Alemu et al: Plasmodium ovale curtisi and Plasmodium ovale wallikeri in North-West Ethiopia. Malaria Journal 2013 12:346.

\section{Submit your next manuscript to BioMed Central and take full advantage of:}

- Convenient online submission

- Thorough peer review

- No space constraints or color figure charges

- Immediate publication on acceptance

- Inclusion in PubMed, CAS, Scopus and Google Scholar

- Research which is freely available for redistribution 